\title{
Organizaciones científicas estudiantiles como semilleros de líderes y gestores de la investigación científica en el Perú: SOCIMEP
}

\section{Scientific student organizations as hotbeds for leaders and managers of scientific investigation in Peru: SOCIMEP}

Correspondencia Aleksandar Cvetkovic Vega aleksandar.famurp@gmail.com presidencia.socimep@gmail.com

Recibido: 26/09/2016 Aprobado: 12/10/2016

Citar como: Cvetkovic-Vega A, Inga-Berrospi F, Mestas C. Organizaciones cientificas estudiantiles como semilleros de líderes y gestores de la investigación cientifica en el Perú: SOCIMEP. Acta Med Peru. 2016;34(1):70-1

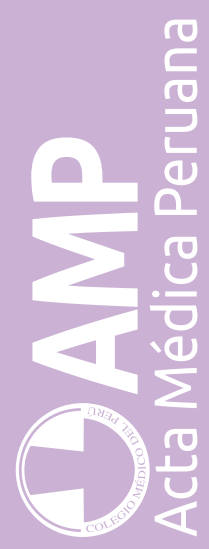

\author{
Aleksandar Cvetkovic-Vega ${ }^{1,2,3, a}$, Fiorella Inga-Berrospi i,4,5,b, Ciro Abel Mestas ${ }^{b}$ \\ 1 Facultad de Medicina Humana, Universidad Ricardo Palma. Lima, Perú. \\ 2 Sociedad Científica Médico Estudiantil Peruana, SOCIMEP. Lima, Perú. \\ 3 Sociedad Científica de Estudiantes de Medicina, Universidad Ricardo Palma. Lima, Perú. \\ 4 Colegio Médico del Perú. Lima, Perú. \\ 5 Federación Latinoamericana de Sociedades Científicas de Estudiantes de Medicina, FELSOCEM. Asunción, Paraguay. \\ a Estudiante de medicina. \\ b Médico cirujano.
}

\section{Sr. Editor:}

Es sabido que la investigación científica es uno de los pilares sobre los que se rigen las universidades para generar conocimientos, al punto que algunos autores la consideran como su función principal ${ }^{[1]}$. Sin embargo, investigar resulta difícil para la mayoría de estudiantes universitarios peruanos por diferentes limitaciones ${ }^{[2]}$ como la falta de valoración del trabajo realizado, ausencia de cultura de publicación, falta de capacitación, desconocimiento de oportunidades, deficiencias en la calidad de la investigación, mínimo apoyo docente y falta de incentivos.

Para subsanar las deficiencias mencionadas y lograr la adquisición de capacidades científicas y publicación, entre otras competencias ${ }^{[3]}$, los estudiantes de medicina han generado espacios académico-científicos que además les permiten disertar y publicar sus aportes científicos en ciencias de la salud. Desde hace 24 años los esfuerzos dedicados para tales fines se vienen organizando y congregando en la Sociedad Científica Médico Estudiantil Peruana (SOCIMEP) que reúne a Sociedades Científicas de facultades de medicina de todo el Perú. Fundada el 27 de agosto de $1992^{[4]}$, inicialmente bajo el nombre de Sociedad Científica Peruana de Estudiantes de Medicina, con el objetivo de fomentar la investigación científica en el pregrado de medicina humana y ser el motor de transformación de la realidad peruana en salud, SOCIMEP actualmente reúne a 40 Sociedades Científicas de Estudiantes de Medicina (SOCIEMS) afiliadas en 18 regiones del Perú y distribuidas en 3 Consejerías Regionales del Norte, Centro y Sur.

Para cumplir con su objetivo, SOCIMEP realiza actividades dedicadas a promover la investigación en salud desde el pregrado en los recursos humanos del país. Las principales iniciativas de fomento de la investigación científica son las Asambleas Generales desde donde se promueve la gestión de la investigación en la etapa de planificación y organización que luego son ejecutadas a través de sociedades científicas locales y regionales; los Campamentos Universitarios de Investigación y Salud $(\text { CUMIS })^{[5]}$ que fomentan la investigación aplicada a la comunidad; las Jornadas Científicas Regionales donde se diserta y discute investigaciones científicas; y finalmente el evento magno de SOCIMEP: el Congreso Científico Nacional de Estudiantes de Medicina (CCN) que en este año celebra su trigésima versión ininterrumpida desde 1987. En ese contexto, los aportes de investigación en salud que se han publicado en 30 libros de resúmenes de los CCN son realmente un legado invalorable de distintas 
generaciones de estudiantes de medicina que han publicado y disertado sus trabajos de investigación en estos eventos. Sin embargo, muchas de estas publicaciones no han sido sistematizadas ni catalogadas adecuadamente.

Actualmente hemos tomado la alternativa de valorar el aporte de SOCIMEP cuantificando las publicaciones en revistas indizadas como el único indicador de la producción científica de las SOCEMs ${ }^{[6]}$. Por ejemplo, el año 2005 se reportó que entre los años 1993 y 2003 hubo 679 artículos de investigación publicados ${ }^{[7]}$. Recientemente, en el año 2015, SOCIMEP alcanzó su récord máximo de publicaciones científicas: 95 publicaciones distribuidas entre 41 cartas al editor, 39 artículos originales y 11 casos clínicos, lo que probablemente signifique uno de los mayores aportes de parte de estudiantes de pregrado al desarrollo de las ciencias de la salud en el Perú.

Para tal fin, los médicos y estudiantes de medicina peruanos, en conjunto, fomentamos la investigación científica dentro de los lineamientos nacionales de investigación y buscamos asociarnos con mentores en investigación, pues a través de modelos y desarrollando investigación es como se aprende a investigar ${ }^{[8]}$. Asimismo, el 25 de setiembre del 2016 en el marco de la Sesión Anual Descentralizada de Comités del Médico Joven se firmó un Acuerdo de Cooperación Institucional entre el Comité Médico Joven del Colegio Médico del Perú y SOCIMEP que busca fortalecer el trabajo con los estudiantes de medicina, futuros médicos jóvenes, a través del desarrollo de líneas de investigación y promoción de la salud; además, se reconoció a SOCIMEP como la organización estudiantil científica nacional de estudiantes de medicina, siendo el Comité Médico Joven la primera institución en el país en reconocer la labor de SOCIMEP por la investigación en el pregrado de medicina en el país. Por lo tanto, hacemos un llamado a las instituciones que promueven la investigación científica a nivel nacional a contribuir y colaborar con el desarrollo de SOCIEMs y en conjunto, mirar hacia el futuro, revalorizando el potencial investigador que los estudiantes peruanos de medicina, agrupados en SOCIMEP pueden brindar a nuestro país desde la visión de un sistema de salud con bases altamente científicas, totalmente integral, universal y equitativo.

\section{REFERENCIAS BIBLIOGRÁFICAS}

1. Cubilla AL. Fines de la Universidad: La investigación como función central. En: Martínez E, Rodríguez JC (Eds.). Universidad en el Paraguay. Desafíos y dilemas. Asunción: Asociación de Estudios Paraguayos - Círculo Paraguayo de Médicos; 2000. p. 71-81.

2. Gutiérrez C, Mayta P. Publicación desde el pregrado en Latinoamérica: importancia, limitaciones y alternativas de solución. Cimel. 2003;8(1):54-60.

3. Mondragón-Cardona Á, Jiménez-Canizales CE, Alzate-Carvajal V. Oportunidades y desarrollo en las sociedades científicas estudiantiles. CIMEL [Internet]. 2012 [citado el 5 de agosto de 2014];17(1):51. Disponible en: http://www.cimel.felsocem.net/ index.php/CIMEL/article/viewArticle/231

4. Alvarado Gamarra AG, Inga Berrospi F. Un reconocimiento al estudiante de medicina en el Perú. Acta Med Peru. 2010;27(4):226.

5. Puescas-Sánchez P, Patiño-Calla K, León-Velándres A, ApolayaSegura M. Importancia de la investigación en atención primaria en salud desde el pregrado de medicina. Revista ANACEM. 2012;6(2):120-1.

6. Rojas-Revoredo V. Las publicaciones en revistas indexadas, único indicador de la producción de las sociedades científicas estudiantiles. CIMEL. 2007;12(1):5-6.

7. Edén Galán, Nadya Manrique, EdgarVillavicencio, EulogioYllatopa Maritrini Peralta, Walter De la Cruz. Producción Científica de los Investigadores del Pregrado de Medicina Humana del Perú, 19932003. CIMEL. 2005;10(1):41-8.

8. Arce-Villavicencio Y, Astuvilca-Cupe J. Grupos Estudiantiles de Investigación: una prioridad en las sociedades científicas estudiantiles de Latinoamérica. CIMEL. 2007;12(2):45-6.

\section{Las ediciones anteriores de Acta Médica Peruana están disponibles en:}

\section{www.scielo.org.pe}

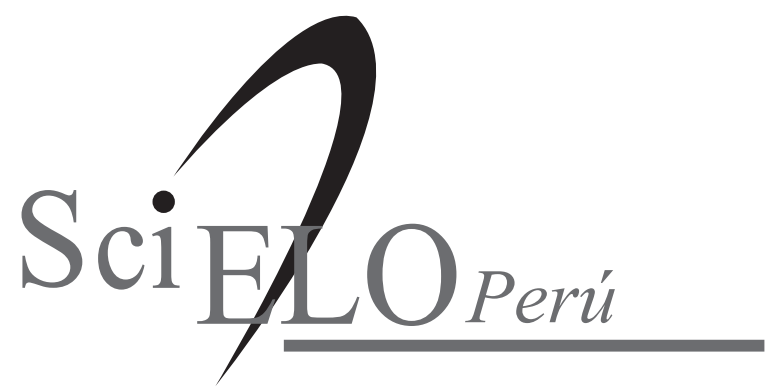

